\title{
AVALIAĈ̣̃O dO ÂNGULO ANO-RETAL POR MEIO DE DEFECOGRAMA EM VOLUNTÁRIAS ASSINTOMÁTICAS NULÍPARAS E MULTÍPARAS*
}

\author{
José Eduardo Mourão Santos ${ }^{1}$, Giuseppe D'Ippolito ${ }^{2}$, Lígia Maria Leme ${ }^{3}$, Adriana Sañudo ${ }^{4}$ \\ David Carlos Shigueoka ${ }^{5}$, Jacob Szejnfeld ${ }^{6}$
}

Resumo OBJETIVO: Fizemos um protocolo para compararmos as medidas dos ângulos ano-retais em três situações diferentes, em voluntárias assintomáticas nulíparas e multíparas. CASUÍSTICA E MÉTODOS: Foram realizados defecogramas em 30 mulheres (15 nulíparas e 15 multíparas), de maio de 1997 a dezembro de 1998, e obtidas incidências radiográficas em perfil do reto após introdução de contraste baritado texturizado: em repouso, durante contração do músculo puborretal e durante a evacuação. Na análise estatística foi utilizada a análise de medidas repetidas. RESULTADOS: A média do ângulo não apresentou diferença significante entre as voluntárias nulíparas e multíparas. 0 ângulo mediu, nas nulíparas, $92,9^{\circ}$ em repouso, $78,8^{\circ}$ durante a contração do músculo puborretal e $117,9^{\circ}$ durante a evacuação, e nas multíparas mediu $94,3^{\circ}$ em repouso, $79,7^{\circ}$ durante a contração do músculo puborretal e $121,4^{\circ}$ durante a evacuação. Foi observada diferença significante entre os ângulos em repouso, durante a contração do músculo puborretal e durante a evacuação. CONCLUSÃO: Não houve diferença significante entre os dois grupos examinados.

Unitermos: Defecografia; Ânus; Reto; Defecação; Pelve; Feminino.

Abstract Measurement of the anorectal angle by defecography in asymptomatic nulliparous and multiparous volunteers.

OBJECTIVE: We developed a protocol to compare the measurements of the anorectal angle in nulliparous and multiparous asymptomatic volunteers in three different situations. SUBJECTS AND METHODS: Defecograms were carried out in 30 women (15 nulliparous and 15 multiparous) from May, 1997 to December, 1998. After rectal administration of texturized barium contrast media, lateral radiographic views of the rectum were obtained at rest, during contraction of the puborectal muscle and during defecation. A repeated measures model was used for the statistical analysis. RESULTS: There were no significant differences between the mean anorectal angles of nulliparous and multiparous volunteers. Measurements were as follows: $92.9^{\circ}$ at rest, $78.8^{\circ}$ during the contraction of the puborectal muscle and $117.9^{\circ}$ during defecation for the nulliparous volunteers; $94.3^{\circ}$ at rest, $79.7^{\circ}$ during the contraction of the puborectal muscle and $121.4^{\circ}$ during defecation for the multiparous volunteers. A significant difference was observed between the anorectal angle measurements at rest, during contraction of the puborectal muscle and during defecation. CONCLUSION: There were no significant differences between the anorectal angle measurements of the two groups of volunteers examined.

Key words: Defecography; Anus; Rectum; Defecation; Pelvis; Female.

\footnotetext{
* Trabalho realizado no Hospital São Paulo - Universidade Federal de São Paulo/Escola Paulista de Medicina (Unifesp/EPM) deral de São Paulo/Escola Paulista de Medicina (Unifesp/EPM)
São Paulo, SP, e no Hospital Universitário da Fundação Universidade Federal de Mato Grosso do Sul (UFMS), Campo Grande, MS.

1. Mestre em Radiologia Clínica pela Unifesp/EPM.

2. Professor Adjunto do Departamento de Diagnóstico por Imagem da Unifesp/EPM.

3. Professora Adjunta da Cadeira de Radiologia do Departamento de Clínica Médica da UFMS

4. Professora do Departamento de Medicina Preventiva da Unifesp/EPM.

5. Doutor em Radiologia Clínica pela Unifesp/EPM, Médico do Departamento de Diagnóstico por Imagem da Unifesp/EPM. 6. Chefe do Departamento de Diagnóstico por Imagem da Unifesp/EPM.

Endereço para correspondência: Dr. José Eduardo Mourão Santos. Rua Guimarães Passos, 415, casa 2, Vila Mariana. São Paulo, SP, 04107-031. E-mail: ze_eduardomourao@terra. com.br

Recebido para publicação em 24/2/2003. Aceito, após revisão, em 11/3/2003.
}

\section{INTRODUÇÃO}

Este trabalho objetivou preencher uma lacuna na literatura nacional a respeito do exame de defecograma e comparar os achados radiográficos obtidos entre nulíparas e multíparas assintomáticas, assunto pouco explorado na literatura mundial.

As primeiras tentativas de estudo dos mecanismos da defecação tiveram início na década de $60^{(1-3)}$. No início da década de 80, Mahieu et al. ${ }^{(4,5)}$ publicaram estudos em que simplificavam os métodos de estudo anteriormente descritos, diminuindo o tempo e o custo do exame e apresentando medidas objetivas da função evacuatória.
A média do ângulo ano-retal (AAR) normal observada pelos autores foi de $92^{\circ}( \pm$ 1,52 EMP) em repouso e de $137^{\circ}( \pm 1,51$ EMP) durante o esforço evacuatório. $\mathrm{O}$ defecograma demonstrou ser mais sensível do que os exames clínicos na detecção e avaliação das desordens da defecação, avaliando indiretamente a alça puborretal pela mensuração do AAR. Este ângulo apresenta importante papel na fisiopatologia das alterações evacuatórias, ajudando na continência fecal, já que ocasiona um retardo na passagem das fezes ${ }^{(6)}$. Quando as medidas do AAR apresentam-se aumentadas durante o repouso, pode ocorrer passagem contínua das fezes para a ampola, sendo 
uma das causas de incontinência, pois a musculatura puborretal parece estar relacionada com a continência fecal $^{(\mathbf{6})}$. O AAR pode não se modificar durante os esforços evacuatórios, como nos casos em que ocorre a contração paradoxal do músculo puborretal, ocasionando dificuldade da passagem do bolo fecal, com sensação de bloqueio à passagem do bolo fecal ${ }^{(7)}$. Dentre as doenças observadas por Mahieu et al. ${ }^{(\mathbf{5})}$, as mais comuns foram a intussuscepção retal, a intussuscepção retal intra-anal, os prolapsos retais redutíveis manual ou espontaneamente, as retoceles e a acentuação da impressão da alça do músculo puborretal.

\section{CASUÍSTICA E MÉTODOS}

O protocolo desta pesquisa foi aprovado pela Comissão de Ética da Universidade Federal de São Paulo/Escola Paulista de Medicina e pela Diretoria Clínica do Hospital Universitário da Fundação Universidade Federal de Mato Grosso do Sul (UFMS). Em obediência às orientações daquela Comissão, foram dadas cartas de informação e obtidos termos de consentimento de cada voluntária.

Trinta voluntárias entraram em nossa casuística, oriundas do Serviço de Cirurgia Geral e do Serviço de Ginecologia e Obstetrícia do Hospital Universitário da UFMS e de voluntárias externas ao hospital, com os dados colhidos no período de maio de 1997 a dezembro de 1998. Foram critérios de inclusão: sexo feminino, sem queixas referentes ao trato gastrintestinal baixo (cólon e reto) ou à defecação, e sem alterações ano-retais ao exame proctológico simplificado, consistindo de inspeção externa e toque retal.

As voluntárias foram divididas em dois grupos. O primeiro grupo foi considerado o grupo controle, sendo constituído por 15 mulheres nulíparas, com somente uma mulher apresentando número de gestações igual a 1 (abortamento espontâneo na sétima semana de gestação). Neste grupo a idade variou entre 15 e 47 anos (média de idade e mediana de 27 anos). O segundo grupo foi formado por 15 mulheres multíparas apresentando número de gestações (G) $\geq 2$ (variação: G2 a G11; média de 3,5) e número de partos $(\mathrm{P}) \geq 2$ (variação: P2 a
P11; média de 3,5). Neste grupo a idade variou entre 25 e 55 anos (média de idade de 35 anos e mediana de 37 anos).

Uma mistura de sulfato de bário com purê de batatas, na proporção de $1 \mathrm{ml}: 1 \mathrm{~g}$ e homogeneizada em liquidificador, foi introduzida no reto $(80 \mathrm{ml})$, seguindo método semelhante ao descrito por Yeong ${ }^{(8)}$. As radiografias foram realizadas em aparelho telecomandado. O assento sanitário utilizado em nossa pesquisa obedeceu aos requisitos descritos por Somers ${ }^{(\mathbf{9 )}}$ : ser radiotransparente, resistente e ter um desenho que permita a obtenção de projeções laterais e frontais. Utilizamos assento de plástico $^{(\mathbf{1 0 , 1 1 )}}$, com pequena câmara pneumática preenchida com água interposta entre a paciente e a base do assento ${ }^{(\mathbf{1 2}-15)}$. Foi utilizada uma mesa de madeira como base para que houvesse uma boa exposição do reto.

Três incidências em perfil do reto foram realizadas em cada paciente, acompanhadas por escopia intermitente: a primeira incidência com a paciente em repouso, a segunda incidência com a paciente contraindo os glúteos e a musculatura puborretal, e a terceira incidência com a paciente realizando esforço evacuatório. O AAR foi mensurado utilizando-se o método des-

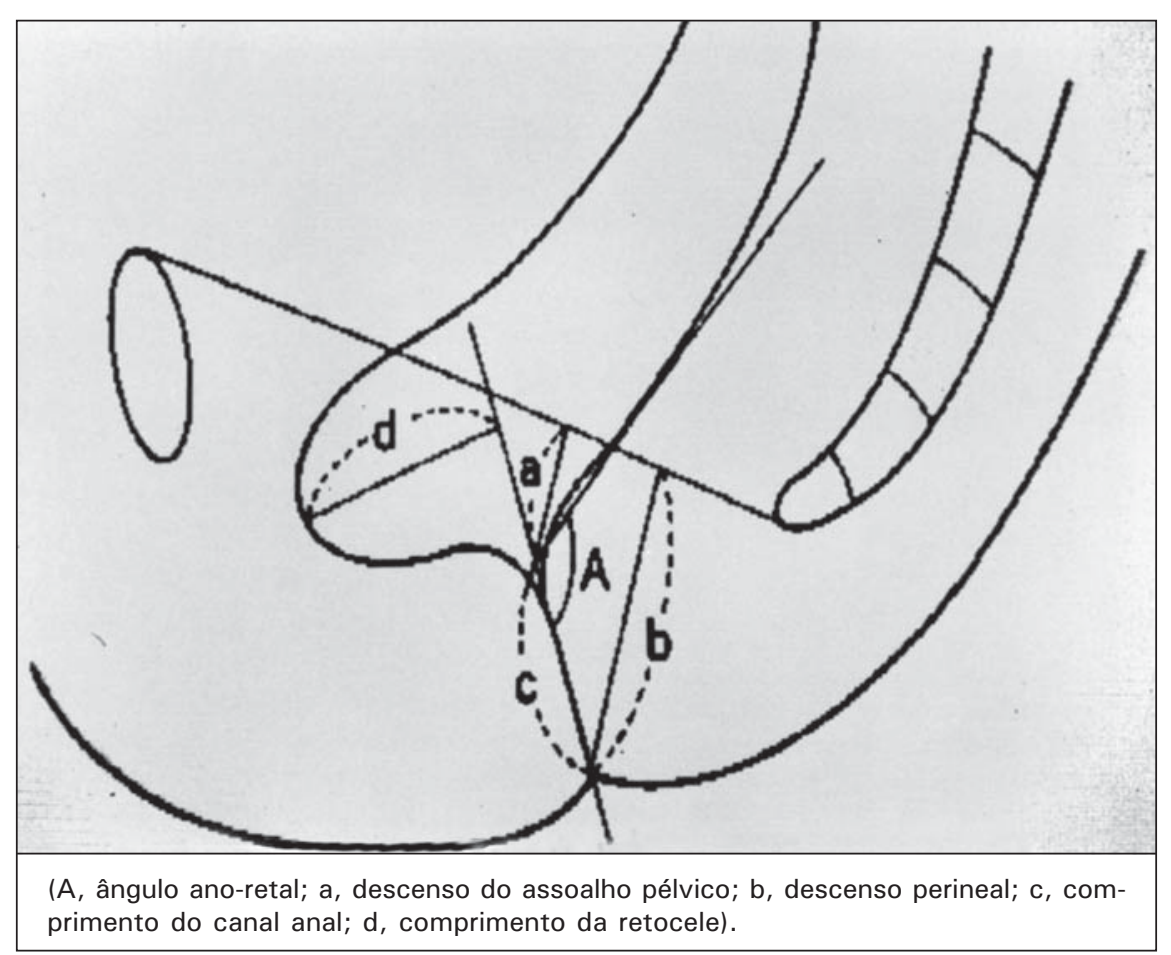

Figura 1. Esquema representativo dos parâmetros anatômicos utilizados no defecograma. crito por Mahieu et al. ${ }^{(4)}$, obtido através da interseção das retas traçadas pelo eixo do canal anal e da parede póstero-inferior do reto (Figura 1).

Como as 30 pacientes foram avaliadas em todas as incidências e as observações puderam ser correlacionadas, foram consideradas mais apropriadas as técnicas de análise de medidas repetidas para o estudo estatístico dos dados ${ }^{(\mathbf{1 6})}$.

O AAR nas mulheres nulíparas variou: em repouso, de $87^{\circ}$ a $103^{\circ}$ (média de $92,9^{\circ}$ ); durante a contração da musculatura glútea e puborretal, de $69^{\circ}$ a $90^{\circ}$ (média de $78,8^{\circ}$ ); em esforço evacuatório, de $108^{\circ}$ a $129^{\circ}$ (média de $117,9^{\circ}$ ). Nas multíparas o AAR variou: em repouso, de $88^{\circ}$ a $99^{\circ}$ (média de $94,3^{\circ}$ ); durante a contração da musculatura glútea e puborretal, de $72^{\circ}$ a $86^{\circ}$ (mé$109^{\circ}$ a $132^{\circ}$ (média de $121,4^{\circ}$ ).

As Figuras 2 e 3 mostram as radiografias do defecograma de uma voluntária nulípara e de uma voluntária multípara, respectivamente.

A Tabela 1 apresenta a média e errospadrão para cada um dos grupos.

\section{RESULTADOS} dia de $79,7^{\circ}$ ); em esforço evacuatório, de 


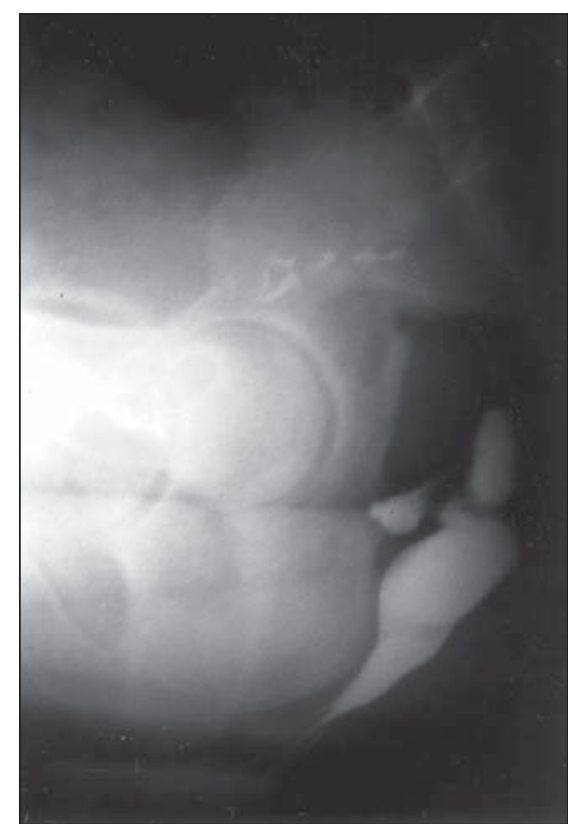

A

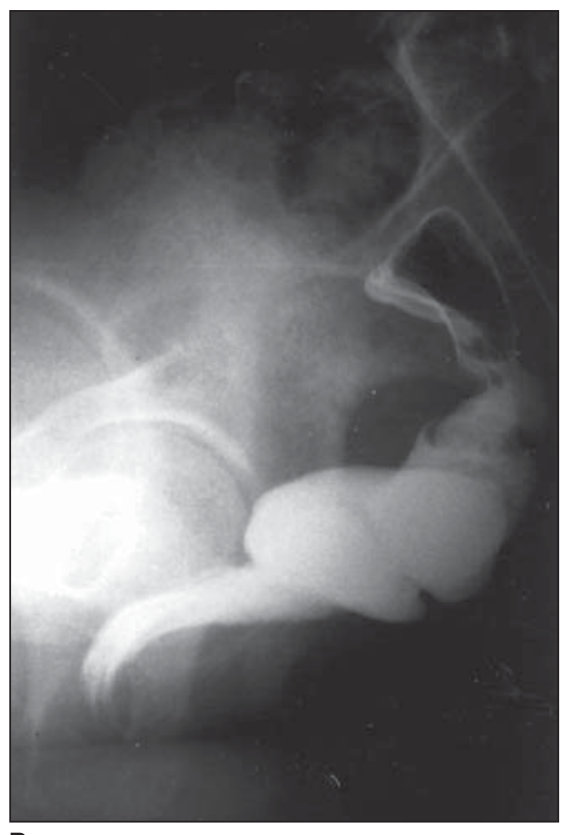

B

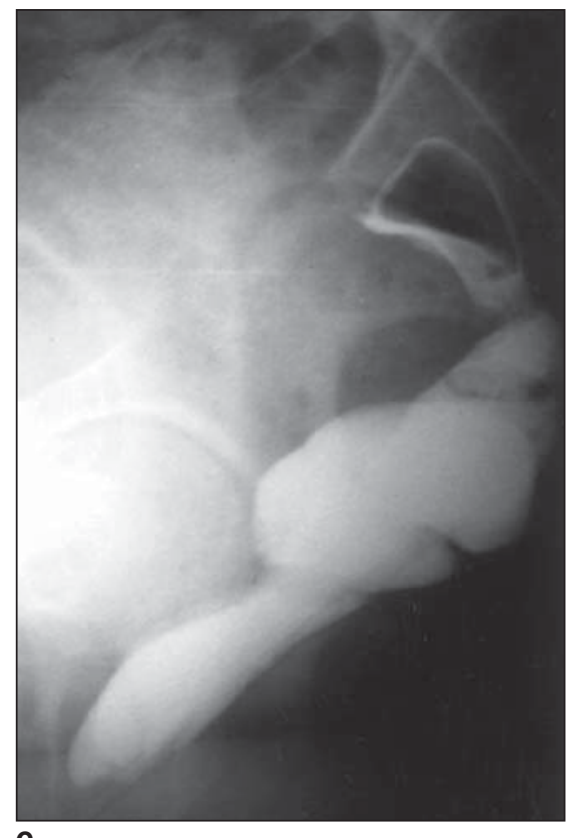

C

Figura 2. Radiografias da voluntária nulípara: em repouso (A), durante a contração da musculatura puborretal e glútea (B) e em esforço evacuatório (C).

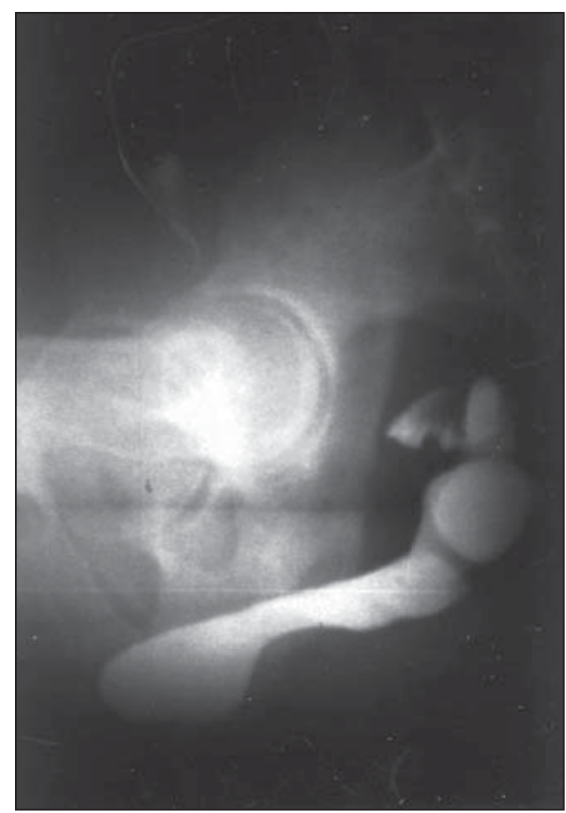

A

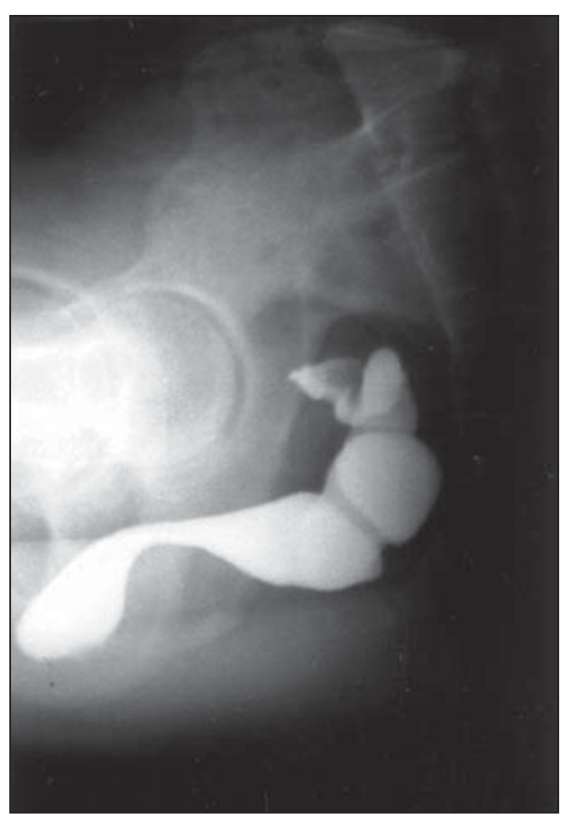

B

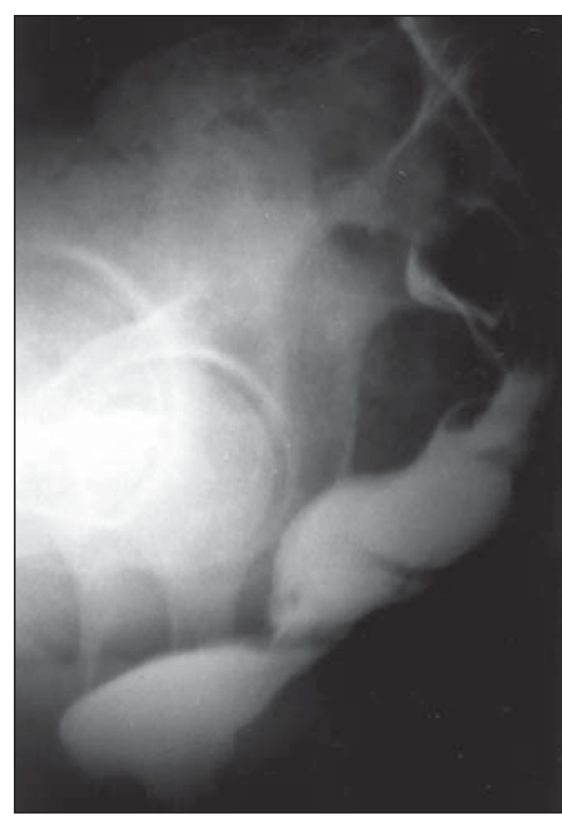

C

Figura 3. Radiografias da voluntária multípara: em repouso (A), durante a contração da musculatura puborretal e glútea (B) e em esforço evacuatório (C).

A Figura 4 apresenta o gráfico de perfil médio para o AAR em cada uma das incidências. Nesse gráfico estão apresentados os resultados observados neste estudo.

O resultado da análise de perfis para o AAR indica que não há efeito significativo da interação incidência $\times$ grupo $(\mathrm{p}=$ $0,3101)$ e de grupo $(p=0,1707)$. Há efeito significativo de incidência $(\mathrm{p}=0,0001)$, ou seja, a variação observada no AAR médio é significativa.

Verificamos, ainda, que existe diferença significativa entre o AAR em repouso e o AAR em contração ( $p=0,0001)$; a diferença média entre o AAR em repouso e o AAR em contração pode ser estimada em $14,3^{\circ}$ (erro-padrão = 0,9). A diferença entre o AAR em repouso e em esforço tam- bém foi significativa ( $\mathrm{p}=0,0001)$; a diferença média entre o AAR em repouso e em esforço pode ser estimada em $26,1^{\circ}$ (erropadrão $=1,2$ ). Também existe diferença significativa entre o AAR em contração e em esforço ( $p=0,0001)$; a diferença média entre o AAR em contração e em esforço pode ser estimada em $-40,4^{\circ}$ (erro-padrão $=1,4)$. 


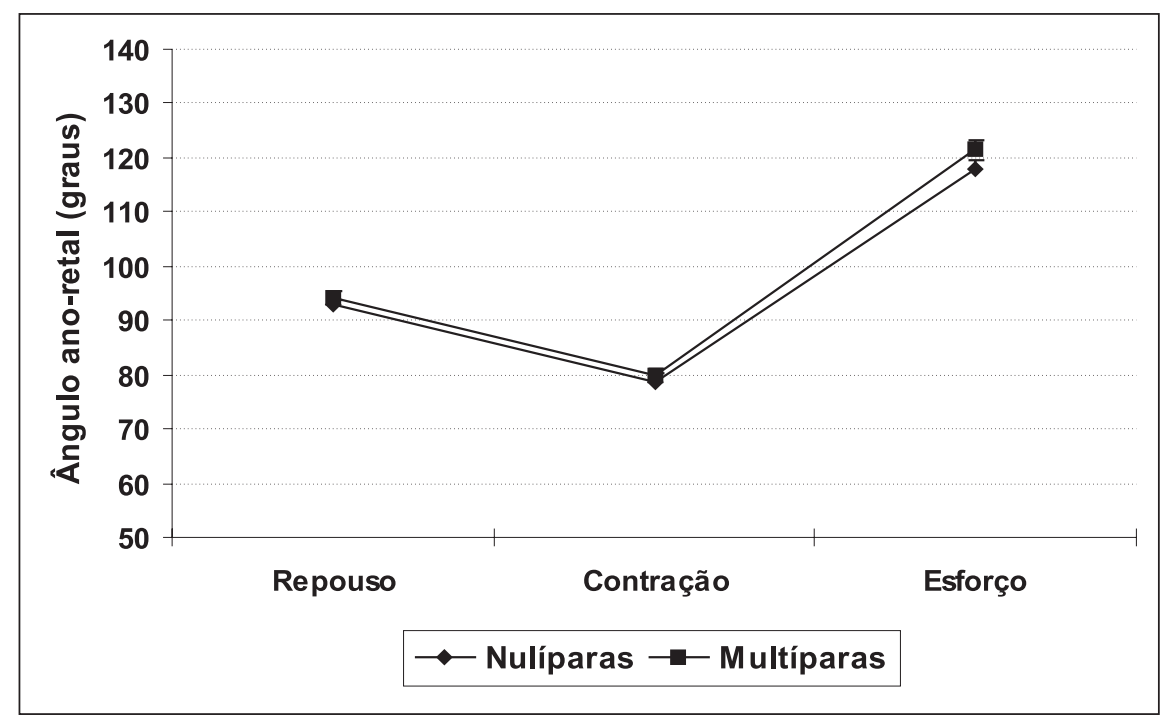

Figura 4. Perfil médio do ângulo ano-retal para cada grupo.

Tabela 1 Média \pm erro-padrão do ângulo ano-retal, em graus, de cada incidência para cada grupo.

\begin{tabular}{|l|c|c|c|}
\hline \multirow{2}{*}{ Grupo } & \multicolumn{3}{|c|}{ Incidência } \\
\cline { 2 - 4 } & Repouso & Contração puborretal e glútea & Esforço evacuatório \\
\hline Nulíparas & $92,9 \pm 1,1$ & $78,8 \pm 1,4$ & $117,9 \pm 1,6$ \\
Multíparas & $94,3 \pm 0,9$ & $79,7 \pm 1,2$ & $121,4 \pm 1,9$ \\
\hline
\end{tabular}

Tabela 2 Classificação das retoceles por tipos, nos grupos de mulheres estudadas, com médias de idade, gestações e paridade nas multíparas.

\begin{tabular}{|c|r|r|c|c|c|c|c|}
\hline \multirow{2}{*}{ Tipos } & \multicolumn{3}{|c|}{ Nulíparas } & \multicolumn{4}{c|}{ Multíparas } \\
\cline { 2 - 7 } & $\mathrm{N}$ & $\%$ & $\begin{array}{c}\text { Média de idade } \\
\text { (anos) }\end{array}$ & $\mathrm{N}$ & $\%$ & $\begin{array}{c}\text { Média de idade } \\
\text { (anos) }\end{array}$ & $\begin{array}{c}\text { Média de gestações } \\
\text { e partos }\end{array}$ \\
\hline I & 2 & 13 & 19 & 5 & 33,33 & 34 & 3,6 \\
II & 6 & 40 & 24 & 5 & 33,33 & 41 & 4,8 \\
III & 7 & 47 & 32 & 5 & 33,33 & 40 & 2,8 \\
IV & 0 & 0 & 0 & 0 & 0 & 0 & \\
\hline & 15 & 100 & & 15 & $\cong 100$ & & \\
\hline
\end{tabular}

Tipos: I, não há retocele mensurável; II, retoceles $<2 \mathrm{~cm}$; III, retoceles de $2-4 \mathrm{~cm}$; IV, retoceles $>4 \mathrm{~cm}$.

A retocele anterior também foi mensurada nas mulheres examinadas, sendo classificada, de acordo com o seu comprimento, em: pequena $(<2 \mathrm{~cm})$, moderada $(2-4$ $\mathrm{cm})$ e grande $(>4 \mathrm{~cm})^{(\mathbf{1 7})}$ (Tabela 2$)$.

Somente $13 \%$ das voluntárias nulíparas não apresentaram retocele. Em $33 \%$ das voluntárias multíparas não foi encontrada evidência radiográfica de retocele. Não se observou nenhum caso de retocele grande (> $4 \mathrm{~cm}$ ) em ambos os grupos. As retoceles classificadas como moderadas foram maioria na casuística encontrada entre as voluntárias nulíparas (47\%), enquanto nas voluntárias multíparas a distribuição foi idade semelhantes. A média de partos foi menor nas voluntárias que apresentaram retocele moderada (média de partos $=3,6$ ) e maior nas que apresentaram retocele pequena (média de partos $=4,8$ ). Alguns autores consideram retoceles abaixo de 2 a $3 \mathrm{~cm}$ sem significado radiográfico ${ }^{(\mathbf{1 8})}$. Duas das voluntárias nulíparas, com idades de 36 e 47 anos, apresentaram descenso do assoalho pélvico acima de $3,5 \mathrm{~cm}^{(\mathbf{1 4})}$. Não foram observadas outras alterações dignas de nota nos demais exames radiográficos estudados.

\section{DISCUSSÃO}

O número de pacientes que apresentam doenças relacionadas à defecação vem aumentando, em virtude de uma melhor conscientização do problema pelos clíni$\cos ^{(\mathbf{1 4})}$. A constipação, uma das maiores queixas de pacientes submetidos ao defecograma, é responsável por quase 2,5 milhões das consultas médicas realizadas nos Estados Unidos a cada ano ${ }^{(\mathbf{1 9})}$. Não há referências a este respeito no Brasil, porém Zeitune $^{(6)}$ comenta, em seu artigo, o aumento da incidência e prevalência da doença no país, oriundo da expectativa de vida cada vez maior da população.

Muitos trabalhos vêm estudando técnicas de exame que possam avaliar o ato evacuatório e as alterações anatômicas e fisiológicas que possam estar relacionadas a sintomas associados a essas alterações. Vários testes procuram avaliar a integridade da musculatura do assoalho pélvico e do esfíncter anal, e da inervação aferente e eferente desta região, como, por exemplo, a manometria ano-retal, a mensuração da sensibilidade retal e da sensibilidade anal, e a eletromiografia. Porém, na área de imagem não há muitas opções de exame que possam auxiliar na detecção de afecções como constipação e incontinência fecal, nem de dor à evacuação ou de sangramento retal. O defecograma tenta ocupar esta lacuna, merecendo muitos estudos nas últimas duas décadas.

Muitos autores criticam o valor da proctografia na seleção de pacientes a serem submetidos a tratamento cirúrgico ${ }^{(20-22)}$. O fato de achados considerados anormais no exame serem encontrados também em pacientes assintomáticos e a falta de consenso 
nas medidas obtidas no defecograma são as causas mais comumente citadas nessas críticas. Não há, no entanto, métodos padrãoouro que sirvam de comparação ${ }^{(23)}$. $\mathrm{Na}$ defesa do método, Karasick e Spettell ${ }^{(\mathbf{2 4})}$ obtiveram acurácia de $83 \%$ em seus exames e Agachan et al. ${ }^{(25)}$ encontraram bons resultados em 744 pacientes submetidos ao defecograma.

Acredita-se que os traumas provocados pelo parto e por histerectomia determinem alterações relacionadas à evacuação. Karasick e Spettell ${ }^{(\mathbf{2 4})}$ confirmam essas crenças em seu trabalho de revisão de 272 mulheres que realizaram defecograma. Eles concluem, ainda, que mulheres que tiveram três ou mais partos têm maior probabilidade de apresentarem incontinência e descenso excessivo do assoalho pélvico do que mulheres que tiveram poucos partos. Em relação às mulheres histerectomizadas, esses autores encontraram uma incidência maior de enteroceles e descenso excessivo do assoalho pélvico. Em nossa casuística não observamos diferença significativa entre as voluntárias nulíparas e as multíparas, e a medida do AAR também não apresentou alterações nos dois grupos estudados, porém as pacientes representadas na pesquisa de Karasick e Spettel ${ }^{(24)}$ são sintomáticas e o trabalho desses autores é retrospectivo.

As médias obtidas em nossa pesquisa podem ser consideradas estatisticamente (técnica de análise de medidas repetidas) como provenientes de uma mesma população; no entanto, poder-se-á observar que houve diferença significativa dentro do mesmo grupo e intergrupos. Neste caso não há como debitar esta variação como sendo oriunda da diferença na escolha do ponto anatômico no qual a medida do ângulo foi baseada. Isto porque a mensuração foi realizada pelo mesmo observador. Esta variação é encontrada também na literatu$\mathrm{ra}^{(\mathbf{4 , 9 , 1 8 , 1 9 , 2 6 - 3 0 )}}$, tendo os diversos autores citados realizado o exame em pacientes assintomáticos e situado suas medidas nos mesmos acidentes anatômicos: eixo do canal anal e parede posterior e inferior do reto (Quadro 1).

Em relação às médias apresentadas pelos autores acima citados, as medidas dos AAR aferidas no presente trabalho apresentam a mesma variabilidade, situando-se

Quadro 1 Medidas do ângulo ano-retal nas diversas incidências, por autores, discriminando o número de pacientes e sexo ou paridade.

\begin{tabular}{|c|c|c|c|c|}
\hline \multirow[b]{2}{*}{ Autor } & \multirow[b]{2}{*}{$\begin{array}{c}\text { № de } \\
\text { pacientes } \\
\text { estudados }\end{array}$} & \multicolumn{3}{|c|}{ Ângulo ano-retal } \\
\hline & & Repouso & $\begin{array}{c}\text { Contração } \\
\text { da musculatura } \\
\text { puborretal }\end{array}$ & $\begin{array}{c}\text { Esforço } \\
\text { evacuatório }\end{array}$ \\
\hline Mahieu et al., $1984^{(4)}$ & 56 (220 34우) & $91,9^{\circ}$ & Não mensurado & $136,7^{\circ}$ \\
\hline Bartolo et al., $1985^{(26)}$ & 35 (10 $100^{\pi} 25$ 우) & $92,5^{\circ}$ & Não mensurado & $111^{\circ}$ \\
\hline Bartram et al., 1988 & 20 (10 $100^{x} 10$ 우) & $94^{\circ}$ & Não mensurado & $113^{\circ}$ \\
\hline Goei et al., 1989(28) & $32\left(190^{\pi} 13 ㅇ ㅜ\right)$ & $104^{\circ}\left(\sigma^{x}\right) 112^{\circ}($ ( $)$ & $81^{\circ}\left(0^{\pi}\right) 86(+\circ)$ & $122^{\circ}\left(\sigma^{\pi}\right) 129^{\circ}($ ( $)$ \\
\hline Shorvon et al., 1989(29) & 48 (250 23우) & $96^{\circ}\left(0^{7}\right) 95^{\circ}$ (早) & $80^{\circ}\left(0^{\top}\right) 71($ ( + $)$ & $98^{\circ}\left(0^{\top}\right) 103^{\circ}$ (ㅇ) \\
\hline Freimanis et al., $1991^{(\mathbf{1 9})}$ & 21 (7 7 o $^{x} 14$ 우) & $98,6^{\circ}$ & Não mensurado & $121,6^{\circ}$ \\
\hline Mezwa et al., 1993(18) & Sem referência & $90^{\circ}$ & Não mensurado & $135^{\circ}$ \\
\hline Somers, $1994^{(9)}$ & Sem referência & $95^{\circ}$ & $28^{\circ}\left(\sigma^{\top}\right) 19^{\circ}($ ( $)$ & $98^{\circ}\left(0^{\top}\right) 103^{\circ}($ ( $)$ \\
\hline Ikenberry et al., $1996^{(30)}$ & 20 (100 10우) & $93^{\circ}\left(0^{7}\right) 89,5^{\circ}$ (ㅇ) & $85^{\circ}\left(0^{\prime \prime}\right) 84,5^{\circ}($ ( $)$ ) & $127^{\circ}\left(\sigma^{\top}\right) 126^{\circ}$ (早) \\
\hline Santos et al., 1999 & 30 (15N 15M) & $93^{\circ}(\mathrm{N}) 94^{\circ}(\mathrm{M})$ & $79^{\circ}(\mathrm{N}) 80^{\circ}(\mathrm{M})$ & $118^{\circ}(\mathrm{N}) 121^{\circ}(\mathrm{M})$ \\
\hline
\end{tabular}

$\mathrm{N}$, nulíparas; $\mathrm{M}$, multíparas.

próximas aos ângulos considerados normais encontrados pela maioria dos pesquisadores $^{(4,9,18,19,26-30)}$.

A importância da avaliação da musculatura puborretal na continência fecal, por meio da mensuração do AAR, não está completamente explicada, porém sabe-se que tem papel importante na fisiologia da defecação, sendo causa de sensação de bloqueio à passagem do bolo fecal, como ocorre no anismo ${ }^{(7)}$.

Há grande controvérsia no que se refere à importância dos achados radiográficos no defecograma, pois muitos dos aspectos visualizados em pacientes sintomáticos podem ser observados em indivíduos normais. Desse modo, as observações e medidas encontradas no defecograma devem ser interpretadas com cautela, não devendo ser o único critério utilizado para a indicação do tratamento.

\section{CONCLUSÃO}

A média do AAR encontrada nas pacientes nulíparas foi de $93^{\circ} \mathrm{em}$ repouso, $79^{\circ}$ durante a contração da musculatura puborretal e glútea e $118^{\circ}$ em esforço evacuatório, e nas multíparas foi de $94^{\circ} \mathrm{em}$ repouso, $80^{\circ}$ na contração da musculatura puborretal e glútea e $121^{\circ}$ em esforço evacuatório.

Houve diferença significante entre os AAR nas diversas incidências.

Não houve diferença significante na média dos AAR nas pacientes nulíparas e multíparas.

\section{REFERÊNCIAS}

1. Burhenne HJ. Intestinal evacuation study: a new roentgenologic technique. Radiology Clinics 1964; 33:79-84.

2. Kerremans R. Radio-cinematographic examination of the rectum and the anal canal in cases of rectal constipation. A radio-cinematographic and physical explanation of dyschezia. Acta Gastroenterol Belg 1968;31:561-70.

3. Broden B, Snellman B. Procidentia of the rectum studied with cineradiography: a contribution to the discussion of causative mechanism. Dis Colon Rectum 1968;11:330-47.

4. Mahieu P, Pringot J, Bodart P. Defecography: I. Description of a new procedure and results in normal patients. Gastrointest Radiol 1984;9:247-51.

5. Mahieu P, Pringot J, Bodart P. Defecography: II. Contribution to the diagnosis of defecation disorders. Gastrointest Radiol 1984;9:253-61.

6. Zeitune JMR. Constipação intestinal no idoso. Rev Bras Med 1991;47(no esp.):15-6,18,20.

7. Roberts JP, Womack NR, Hallan RI, Thorpe AC, Williams NS. Evidence from dynamic integrated proctography to redefine anismus. Br J Surg 1992; 79:1213-5.

8. Yeong KY. Defecating proctography: local experience. Ann Acad Med Singapore 1993;22:684-7.

9. Somers S. Evacuation proctography. In: Gore RM, Levine MS, Laufer I, eds. Textbook of gastrointestinal radiology. Philadelphia: WB Saunders, 1994 1041-50.

10. Ginai AZ. Evacuation proctography (defecography). A new seat and method of examination. Clin Radiol 1990;42:214-6.

11. Rafert JA, Lappas JC, Wilkins W. Defecography: techniques for improved image quality. Radiol Technol 1990;61:368-73.

12. Goei R, Baeten C, Arends JW. Solitary rectal ulcer syndrome: findings at barium enema study and defecography. Radiology 1988;168:303-6.

13. Hyland GJ. Defaecating video proctography. J Audiov Media Med 1988;11:91-3.

14. Mahieu PHG. Defecography. In: Margulis AR, Burhenne HJ, eds. Alimentary tract radiology. 4th ed. St. Louis: CV Mosby, 1989:933-41.

15. Felt-Bersma RJ, Luth WJ, Janssen JJ, Meuwissen SG. Defecography in patients with anorectal disor- 
ders. Which findings are clinically relevant? Dis Colon Rectum 1990;33:277-84.

16. Singer JM, Andrade DF. Analysis of longitudinal data. In: Rao CR, Sen PK, eds. Handbook of statistics. Amsterdam: Elsevier, 1999; vol. 17.

17. Yang XM, Partanen K, Farin P, Soimakallio S. Defecography. Acta Radiol 1995;36:460-8.

18. Mezwa DG, Feczko PJ, Bosanko C. Radiologic evaluation of constipation and anorectal disorders. Radiol Clin North Am 1993;31:1375-93.

19. Freimanis MG, Wald A, Caruana B, Bauman DH. Evacuation proctography in normal volunteers. Invest Radiol 1991;26:581-5.

20. Wald A, Caruana BJ, Freimanis MG, Bauman DH, Hinds JP. Contributions of evacuation proctography and anorectal manometry to evaluation of adults with constipation and defecatory difficulty. Dig Dis Sci 1990;35:481-7.
21. Nielsen MB, Buron B, Christiansen J, Hegedüs V. Defecographic findings in patients with anal incontinence and constipation and their relation to rectal emptying. Dis Colon Rectum 1993;36:806-9.

22. Ott DJ, Donati DL, Kerr RM, Chen MYM. Defecography: results in 55 patients and impact on clinical management. Abdom Imaging 1994;19:349-54.

23. Klauser AG, Ting KH, Mangel E, Eibl-Eibesfeldt B, Müller-Lissner SA. Interobserver agreement in defecography. Dis Colon Rectum 1994;37:1310 6.

24. Karasick S, Spettell CM. The role of parity and hysterectomy on the development of pelvic floor abnormalities revealed by defecography. AJR 1997; 169:1555-8.

25. Agachan F, Pfeifer J, Wexner SD. Defecography and proctography. Results of 744 patients. Dis Colon Rectum 1996;39:899-905.
26. Bartolo DCC, Roe AM, Virjee J, Mortensen NJ. Evacuation proctography in obstructed defaecation and rectal intussusception. Br J Surg 1985;72 Suppl::S111-6.

27. Bartram CI, Turnbull GK, Lennard-Jones JE. Evacuation proctography: an investigation of rectal expulsion in 20 subjects without defecatory disturbance. Gastrointest Radiol 1988;13:72-80.

28. Goei R, van Engelshoven J, Schouten H, Baeten C, Stassen C. Anorectal function: defecographic measurement in asymptomatic subjects. Radiology 1989;173:137-41.

29. Shorvon PJ, McHugh S, Diamant NE, Somers S, Stevenson GW. Defecography in normal volunteers: results and implications. Gut 1989;30:1737-49.

30. Ikenberry S, Lappas JC, Hana MP, Rex DK. Defecography in healthy subjects: comparison of three contrast media. Radiology 1996;201:233-8. 